\title{
A Single Institutional Analysis of Systemic Therapy for Unresectable or Recurrent Small Bowel Adenocarcinoma
}

\author{
SHUNICHIRO MAKINO*, HIDEKAZU TAKAHASHI*, NAOTSUGU HARAGUCHI, \\ JUNICHI NISHIMURA, TAISHI HATA, CHU MATSUDA, MASAKAZU IKENAGA, KOHEI MURATA, \\ HIROFUMI YAMAMOTO, YUICHIRO DOKI, MASAKI MORI and TSUNEKAZU MIZUSHIMA \\ Department of Gastroenterological Surgery, Graduate School of Medicine, \\ Osaka University, Suita, Japan;
}

\begin{abstract}
Background: Small bowel adenocarcinoma (SBA) is rare. For unresectable or recurrent SBA, systemic treatment is the only potentially promising option. Patients and Methods: This retrospective study included 13 patients with SBA treated at our Institute. All patients received fluorouracil- or fluoropyrimidine-based regimens. We analyzed associations between the progression-free interval (PFI) and therapeutic regimen $(n=17)$ and patient characteristics. Results: The 5-year overall survival of patients with SBA was $23.5 \%$. The 5-year relapse-free survival of patients with curatively resected (RO or R1) SBA $(n=6)$ was $45 \%$. Patients with unresectable or recurrent $S B A$ $(n=10)$ had a median overall survival time of 28.0 months. No factor was significantly associated with PFI, except the number of treatment regimens required: patients that received only one line of treatment survived significantly longer than those receiving multiple lines. Conclusion: Fluorouracil- or fluoropyrimidine-based regimens might effectively treat unresectable or recurrent SBA. We did not investigate any additive agents that might have increased efficacy of these regimens.
\end{abstract}

Although the small bowel occupies more than two-thirds of the length of the digestive system, small bowel malignancy occurs rarely. Small bowel malignancies are estimated to represent $0.1-0.3 \%$ of total malignancies and $1-3 \%$ of total

\footnotetext{
*These Authors contributed equally to this study.

Correspondence to: Hidekazu Takahashi, MD, Ph.D., Department of Gastroenterological Surgery, Graduate School of Medicine, Osaka University, 2-2, Yamadaoka, Suita, Osaka, 565-0871, Japan. Tel: +81668793251, Fax: +81668793259,e-mail; htakahashi@ gesurg.med.osaka-u.ac.jp
}

Key Words: Unresectable, recurrent, small bowel adenocarcinoma, chemotherapy. gastrointestinal adenocarcinomas $(1,2)$. Histologically, among all small bowel malignant tumours, carcinoid tumours, thought to be relatively slow-growing neoplasias, are the most common, and adenocarcinomas are the second most common (3-7).

Like other malignancies of the gastrointestinal tract, the standardized treatment strategy for localized small bowel malignancies is surgical resection. Small bowel adenocarcinoma (SBA) is considered to be biologically more aggressive than carcinoids. The selected treatment for SBA is surgical resection with regional lymph node dissection. Generally, however, it is difficult to diagnose patients with SBA at an early stage due to the lack of established screening methods, such as colon endoscopy screening for colorectal cancer. Future technologies are in development, including capsule endoscopy (8) and double-balloon endoscopy (9). However, currently, a large proportion of patients are diagnosed with SBA at an advanced stage, when symptoms include abdominal pain, intestinal obstruction, bleeding, or bowel perforation. Indeed, 39\% of patients with SBA have lymph node metastasis (stage III) and 35\% have distant metastasis (stage IV). These proportions of late-stage diagnoses are relatively larger than those found for other common malignancies (10).

Systemic treatment is considered to be the only potentially promising option for controlling unresectable or recurrent SBA. Nevertheless, there is currently no established chemotherapy protocol for unresectable or recurrent SBA. Consequently, in daily clinical practice, chemotherapy regimens for treating unresectable or recurrent SBA are based on regimens for treating metastatic colorectal cancer (11). Although the efficacy of cytotoxic agents for SBA has not been verified in a randomized controlled trial, the results of several retrospective studies have suggested that survival was prolonged in patients that underwent chemotherapy with fluoropyrimidine (FP) or fluorouracil (5-FU) combined with oxaliplatin (12-14). However, few studies have reported on the use of biological agents for treating unresectable or recurrent SBA. 


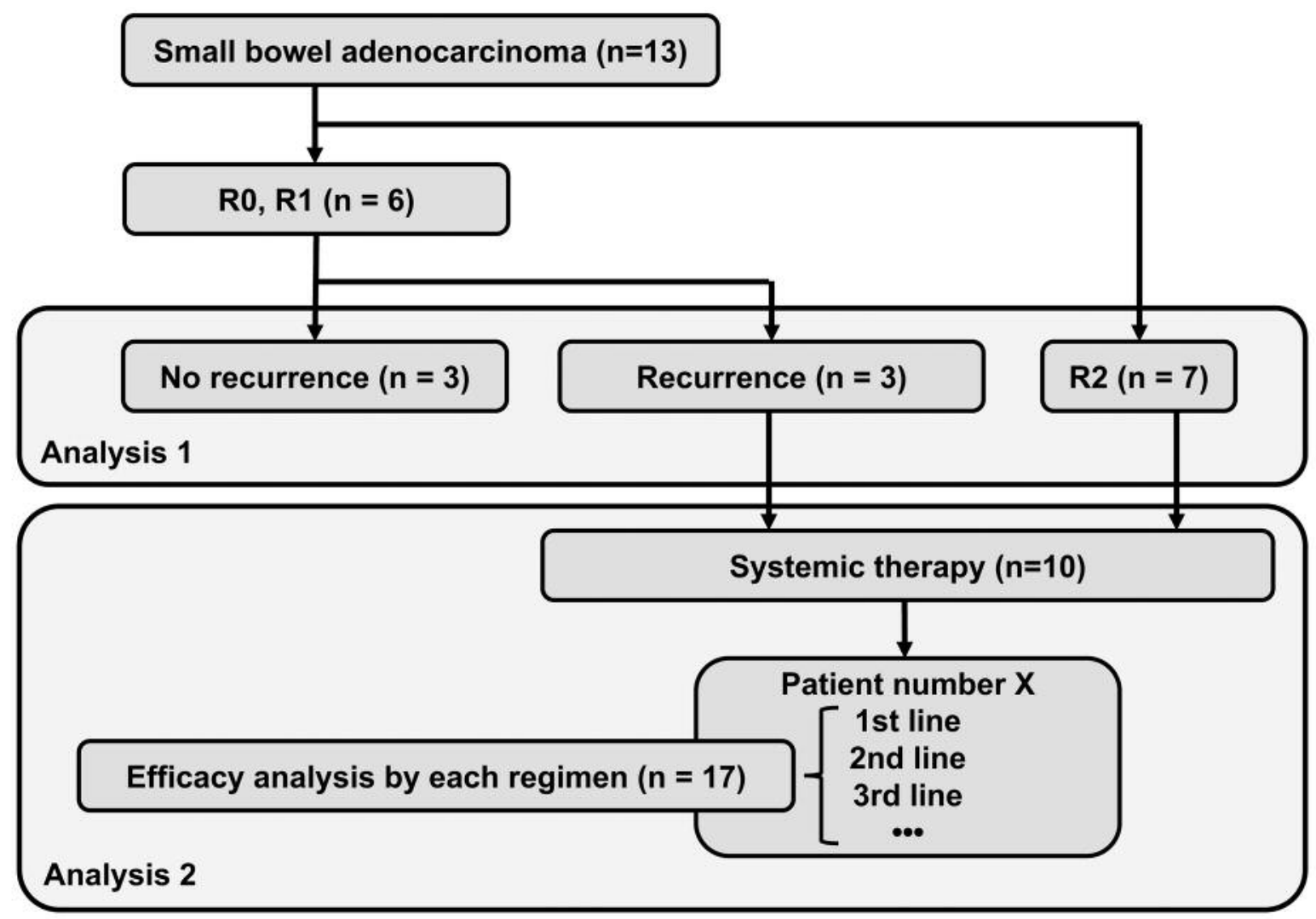

Figure 1. Flowchart of the patient grouping process for the two analyses of this study.

The aims of this study were due to the rarity of SBA, to confirm that the clinicopathological features of SBA in this study population were similar to those previously reported for SBA, and to clarify the outcome of systemic therapy with cytotoxic agents/biological-targeted agents for treating unresectable or recurrent SBA.

\section{Patients and Methods}

Patient selection. The present retrospective study was approved by our Institutional Review Board (approval number 15144). We retrospectively analysed data retrieved from a database of all patients that underwent surgical resections for SBA at Osaka University Hospital (Osaka, Japan) from January 2006 to September 2016. All patients with SBA were diagnosed based on pathological findings.

Analysis 1: Validation of study population. Preoperative staging included carcinoembryonic antigen (CEA) determination and a thoraco-abdomino-pelvic computed tomographic scan. For this study, we reassessed all prognostic factors for SBA found in the literature, including patient characteristics, primary stage, according to the 2010 American Joint Committee on Cancer (AJCC) staging system (15), preoperative carcinoembryonic antigen (CEA) level, and long-term outcomes.
Analysis 2: Analysis of efficacy of therapeutic agents. Of the 13 included patients, six had undergone resections with no residual tumour (R0, R1), and seven incomplete resections or had severe metastases, which were diagnosed as unresectable lesions, due to surgical inaccessibility or severe pathology (R2). Of the six patients with R0 or R1 resection, three had no recurrence, and three had recurrence (two with $\mathrm{R} 0$ and one with $\mathrm{R} 1$ resection). Ten out of 13 patients had unresectable or recurrent SBA (Figure 1). Among these 10 patients, 17 systemic therapy regimens were performed, with sequential treatments in some patients. We analysed data grouped by the therapeutic regimen; the endpoint was the progression-free interval (PFI). PFI was defined as the period during and after treatment in which a participant was living with disease that did not worsen.

Statistical analysis. Statistical differences between the groups were analysed with the Student's $t$-test for continuous variables, the Chisquare test for other variables, and the log-rank test for the PFI. A $p$-value of 0.05 was taken to indicate statistical significance. All statistical analyses were performed with JMP Pro, Version 12 (SAS Institute Inc., Cary, NC, USA).

\section{Results}

Validation of study population. Table I shows the clinicopathological characteristics of the 13 included patients. Seven patients (54\%) were male. The average age 
Table I. Clinicopathological features and treatments of patients with small bowel adenocarcinoma (SBA).

\begin{tabular}{lcc}
\hline Characteristic & $\begin{array}{c}\text { No. of patients } \\
\text { (total=13) }\end{array}$ & $\%$ \\
\hline Male & 7 & $54 \%$ \\
Age, years (mean \pm SD) & $58.3 \pm 12.5$ & \\
Location of primary tumour & & \\
$\quad$ Jejunum & 8 & $62 \%$ \\
Ileum & 5 & $38 \%$ \\
Histological findings & & \\
$\quad$ Well-differentiated & 5 & $38 \%$ \\
Moderately differentiated & 5 & $38 \%$ \\
Poorly differentiated & 2 & $15 \%$ \\
Mucinous & 1 & $8 \%$ \\
AJCC stage & & \\
I & 0 & $0 \%$ \\
II & 4 & $31 \%$ \\
III & 2 & $15 \%$ \\
IV & 7 & $54 \%$ \\
Resection & & \\
R0 & 5 & $38 \%$ \\
R1 & 1 & $8 \%$ \\
R2 & 7 & $54 \%$ \\
Median OS (months) & & 42.7 \\
Median DFI & & ND \\
Mean preoperative CEA ( \pm SD), $\mathrm{ng} / \mathrm{ml}$ & & $2.5 \pm 2.3$ \\
Adjuvant chemotherapy & & \\
Yes & 1 & $92 \%$ \\
No & 12 & \\
\hline
\end{tabular}

AJCC: American Joint Committee on Cancer; OS: overall survival; DFI: disease-free interval; CEA: carcinoembryonic antigen; ND: not determined. aValues indicate the number of patients, unless noted otherwise.

was $58.3 \pm 12.5$ year-old. Five patients underwent radical surgery with lymph node dissection (R0) as extensive as possible. Seven patients had metastases in other organs and peritoneal dissemination (stage IV); these patients underwent palliative surgery (R2).

The 5-year survival rate was $23.5 \%$ for the whole patient group, and the median survival time was 42.7 months (Figure 2A). The 5-year relapse-free survival rate was $45 \%$ among patients that underwent curative resection (R0 or R1; Figure 2B).

Analysis of the efficacy of therapeutic agents. For 10 patients with unresectable or recurrent SBA, 17 systemic therapy regimens were performed sequentially (Table II). Among these 10 patients, the median overall survival time was 28.0 months, starting from the first administration of systemic therapy (Figure 3). All patients received FU- or FP-based regimens (Table II). Table III summarizes the results of an analysis of factors that were potentially associated with the PFI, including each regimen. Patient age, gender, primary
Table II. Therapeutic regimens analysed for efficacy in patients with small bowel adenocarcinoma (SBA) (analysis 2).

\begin{tabular}{lcc}
\hline Patient no & Treatment line & $\begin{array}{c}\text { Regimen for treating } \\
\text { unresectable or recurrent SBA }\end{array}$ \\
\hline 1 & $1 \mathrm{st}$ & XELOX \\
2 & $1 \mathrm{st}$ & XELOX+BV \\
3 & $1 \mathrm{st}$ & XELOX+BV \\
3 & $2 \mathrm{nd}$ & FOLFIRI+BV \\
4 & $1 \mathrm{st}$ & XELOX \\
4 & $2 \mathrm{nd}$ & FOLFOLI+BV \\
5 & $1 \mathrm{st}$ & Xeloda \\
7 & $1 \mathrm{st}$ & XELOX \\
7 & $2 \mathrm{nd}$ & IRIS \\
8 & $1 \mathrm{st}$ & FOLFOLI+BV \\
8 & $2 \mathrm{nd}$ & FOLFOX6+BV \\
8 & $3 \mathrm{rd}$ & Cape+BV \\
9 & $1 \mathrm{st}$ & mFOLFOX6+BV \\
10 & $1 \mathrm{st}$ & TS-1 \\
10 & $2 \mathrm{nd}$ & FOLFOX6+BV \\
11 & $1 \mathrm{st}$ & mFOLFOX6 \\
11 & $2 \mathrm{nd}$ & FOLFILI \\
\hline
\end{tabular}

XELOX: Capecitabine/oxaliplatin; FOLFIRI: irinotecan/leucovorin (LV)/fluorouracil (FU); IRIS: irinotecan/TS1; FOLFOX: oxaliplatin/5FU/LV; BV: bevacizumab; Cape: capecitabine.

tumour location, time of emergence of the target lesions, location of target lesion, and agents used in each regimen had no significant impact on the PFI in this study. Only the number of treatment lines applied had a significant effect on the PSI for unresectable or recurrent SBA: patients that received only one line of treatment survived significantly longer than those that received two or three lines of treatment.

\section{Discussion}

Primary SBA is reported to comprise $0.1-1 \%$ of all primary gastrointestinal malignancies $(1,2,16)$. In the United States, 8,070 new cases and 1,150 deaths were related to small bowel adenocarcinoma in 2012 (17). In Japan, 1250 deaths were related to small bowel adenocarcinoma in 2014 (18). The average age of diagnosis is 65 years for small bowel malignancy and 67-68 years for adenocarcinoma and carcinoid $(19,20)$. SBA is slightly more prevalent in males than in females (1.5:1) (3). According to the National Cancer Database (NCDB), in the United States, the 5-year survival rates for SBA in $1985-1995$ were $65 \%$ for those with stage I disease, $48 \%$ for stage II, $35 \%$ for stage III, and $4 \%$ for stage IV (3). Among patients with SBA, 39\% had lymph node metastases (stage III) and 35\% had distant metastases (stage IV). These proportions were relatively larger than the proportions of metastases among patients with other common 
A

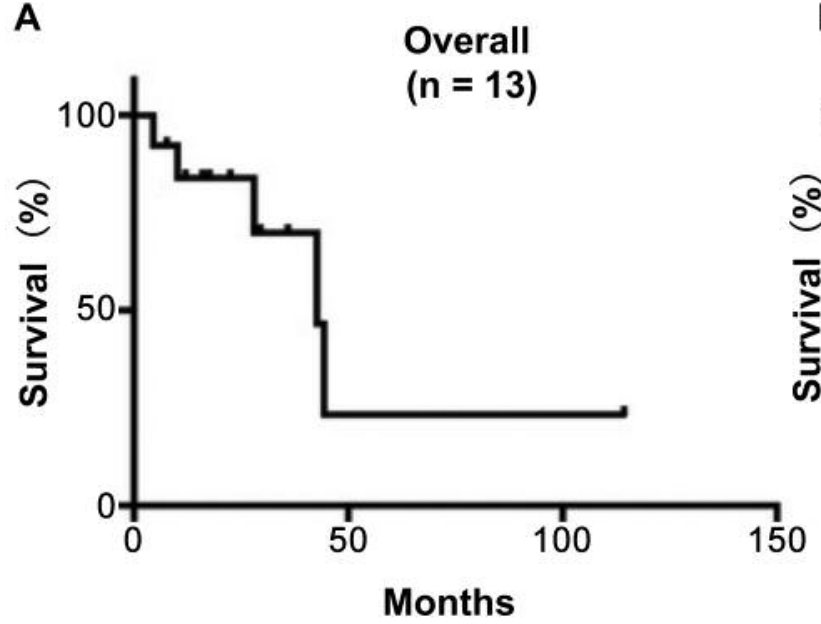

B

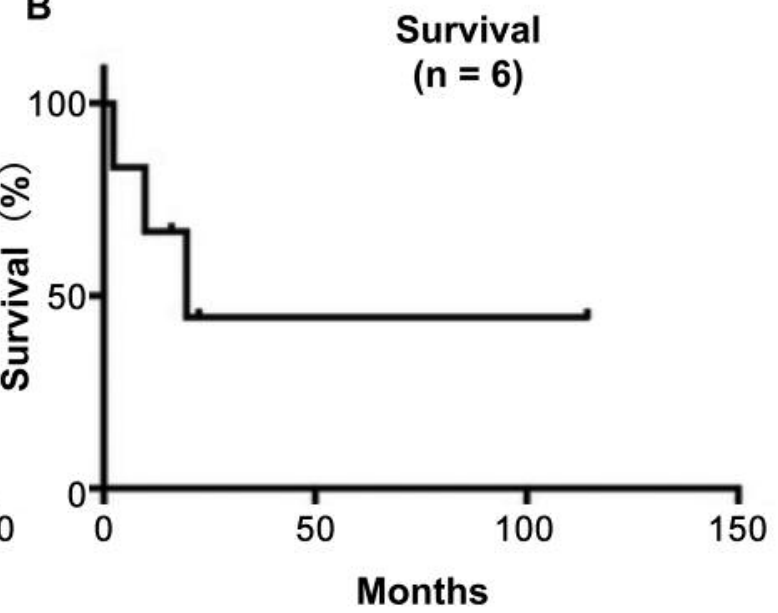

Figure 2. Survival of patients treated for small bowel adenocarcinoma (SBA). A: Kaplan-Meier plot illustrating the overall survival of patients with SBA (n=13). The median overall survival time was 42.7 months. B: Kaplan-Meier plot illustrating the relapse-free survival of patients with curatively resected ( $R 0$ or $R 1$ ) SBA ( $n=6)$. The median relapse-free survival time was not determined.

malignancies (10). In this study, we found (Table I) that the proportion of patients with stages III or IV disease was $69 \%$, and the proportion of patients with stage IV was 54\%. The proportion of patients with advanced SBA was larger than that of previous reports; this indicates that the patients we analysed represented a special population, which was probably due to the fact that our institute was a tertiary hospital in this area.

The basic treatment for small bowel malignancy is radical surgical resection. Depending on the degree of progression, a curative resection requires a $5-10 \mathrm{~cm}$ separation between the intestinal tract and the tumour. This separation provides space for dissection of the intestinal tract, followed by partial resection of the small bowel, and the dissection of regional lymph nodes, as extensively as possible. We found that after a curative resection, the 5-year relapse-free survival rate was $45 \%$ (Figure 2B).

In a previous study conducted in 13 institutes in France, patients with unresectable or recurrent SBA $(n=93)$ received cytotoxic agents for treating advanced SBA. Patients that received FOLFOX [oxaliplatin/5-fluorouracil (5-FU)/ leucovorin] had a better long-term prognosis than those who received other regimens (21). Among the 48 patients that received FOLFOX, the mean progression-free survival was 6.9 months and the overall survival was 17.8 months (13). Similarly, phase II trials were conducted in China to test modified FOLFOX4 therapy for treating unresectable and advanced SBA. That study included 33 patients at three facilities in China. The response rate was $48.5 \%$ (3\% complete response and $45.5 \%$ partial responses); the median time to progression was 7 months, and the median overall survival was

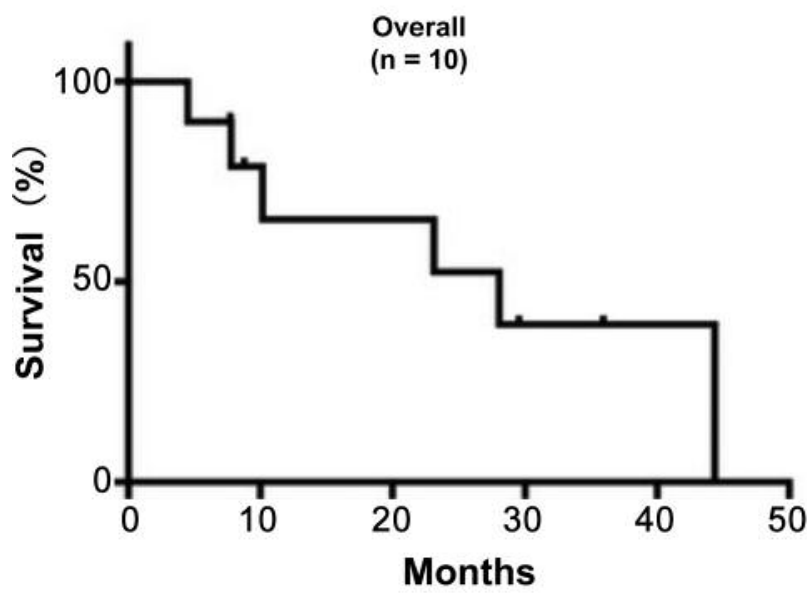

Figure 3. Survival of patients with unresectable or recurrent small bowel adenocarcinoma (SBA) treated with systemic therapy. Kaplan-Meier plot illustrating the overall survival of patients $(n=10)$, starting after the first administration of systemic therapy. Median overall survival time was 28.0 months.

15.2 months (22). Pathologically complete responses were also reported in another study with 5-FU-based chemoradiotherapy administered before surgery (23). Based on a review of 18 articles, Raghav and colleagues recommended a FOLFOX regimen with 5-FU for treating unresectable small bowel cancer (24). In the present study, the proportion of advanced SBA cases was larger than the proportions previously reported; consequently, in contrast to previous reports, we did not find that any individual therapeutic regimen provided an advantage 
Table III. Analysis of factors that might influence the progression-free interval (PFI) after treatment of patients with small bowel adenocarcinoma $(S B A)$.

\begin{tabular}{lccc}
\hline Characteristic & Subgroup & No. of patients & $p$-Value for PFI \\
\hline Patient & Age: $<65$ years/ $\geq 65$ years & $9 / 8$ & 0.35 \\
& Male/female & $8 / 9$ & 0.06 \\
Tumour & Primary location: jejunum/ileum & $10 / 7$ & 0.42 \\
& Synchronous/metachronous & $12 / 5$ & 0.28 \\
& Target lesion: H/N/P/L & $2 / 4 / 6 / 5$ & 0.17 \\
Regimen & Line: 1 st/2nd/3rd & $10 / 6 / 1$ & 0.0003 \\
& 5-FU or FP: With/without & $17 / 0$ & ND \\
& Oxaliplatin: With/without & $9 / 8$ & 0.99 \\
& Irinotecan & $11 / 6$ & 0.37 \\
& Bevacizumab & $9 / 8$ & 0.66 \\
\hline
\end{tabular}

H: Liver metastasis; N: lymph node metastasis; P: peritoneal dissemination; L: local recurrence; FU: fluorouracil; FP: fluoropyrimidine; ND: not determined.

over the others. However, the median survival and relapse-free survival rates were higher than those of previous reports. Our patients with unresectable or recurrent SBA $(n=10)$ had a median overall survival time of 28.0 months, starting after the first administration of systemic therapy (Figure 3). A potential explanation for the good prognosis found in our study could be that our patients were, on average, younger (Table I) than those in previous reports; moreover, our patients were more tolerant of the adverse effects of cytotoxic agents than patients in previous reports.

A recent international phase III clinical trial (NO 16966) compared bevacizumab or placebo in combination with FOLFOX or CAPOX (capecitabine/oxaliplatin) as a first-line treatment for patients with metastatic colorectal cancer. The bevacizumab-treated group experienced significantly superior progression-free survival over the placebo-treated group or (hazard ratio $=0.83 ; p=0.00239)(25)$. However, the median progression-free survival (9.4 vs. 8 months, respectively) was clearly shorter than that recorded in the present study (28 months; Figure 3 ). The results from our analysis of potential associations with the PFI (analysis 2) showed that no regimen, including FU, FP, and the bevacizumab combination regimens, had any significant effect on PFI. In fact, the number of treatment lines applied was the only factor that significantly had an impact on the PFI of patients with unresectable or recurrent SBA (Table III). Patients that received only one line of treatment survived significantly longer than those who received two or three lines of treatment.

The present study had several limitations. Firstly, our institute is only a single, specialized, tertiary hospital; thus, the patient population was different from typical patient populations. Secondly, this study was conducted with a relatively small number of patents. Thirdly, the study design was retrospective. Fourthly, the study period was too long for treatment quality to be equivalent throughout. However, our results might foster further investigations. Future studies involving a larger number of patients are required to confirm the validity of these findings.

\section{Conflicts of Interest}

The Authors declare they have no conflict of interest in regard to this study.

\section{References}

1 Matsuo S, Eto $\mathrm{T}$ and Tsunoda T: Small bowel tumors: an analysis of tumor-like lesions, benign and malignant neoplasms. Eur J Surg Oncol 20: 47-51, 1994

2 Siegel RL, Miller KD and Jemal A: Cancer statistics, 2016. CA Cancer J Clin 66: 7-30, 2016.

3 Bilimoria KY, Bentrem DJ, Wayne JD, Ko CY, Bennett CL and Talamonti MS: Small bowel cancer in the United States: changes in epidemiology, treatment and survival over the last 20 years. Ann Surg 249: 63-71, 2009.

4 Hatzaras I, Palesty JA, Abir F, Sullivan P, Kozol RA, Dudrick SJ and Longo WE: Small-bowel tumors epidemiologic and clinical characteristics of 1260 cases from the Connecticut Tumor Registry. Arch Surg 142: 229-235, 2007.

5 JA D, Burt RW, Vargas H and McWhorter WP: Small bowel cancer: epidemiological and clinical characteristics from a population-based registry. Am J Gastroenterol 89: 699-701, 1994.

6 Garcia Marcilla JA, Sanchez Bueno F, Aguilar J and Parrilla Paricio P: Primary small bowel malignant tumors. Eur J Surg Oncol 20: 630-634, 1994.

7 Weiss NS and Yang CP: Incidence of histologic types of cancer of the small intestine. J Natl Cancer Inst 78: 653-656, 1987.

8 Pennazio M, Spada C, Eliakim R, Keuchel M, May A, Mulder CJ, Rondonotti E, Adler SN, Albert J, Baltes P, Barbaro F, Cellier C, Charton JP, Delvaux M, Despott EJ, Domagk D, Klein A, McAlindon M, Rosa B, Rowse G, Sanders DS, Saurin JC, 
Sidhu R, Dumonceau JM, Hassan C and Gralnek IM: Smallbowel capsule endoscopy and device-assisted enteroscopy for diagnosis and treatment of small-bowel disorders: European Society of Gastrointestinal Endoscopy (ESGE) Clinical Guideline. Endoscopy 47: 352-376, 2015.

9 Lipka S, Rabbanifard R, Kuma A and Brady P: Single versus Double Balloon Enteroscopy for Small Bowel Diagnostics A Systematic Review and Meta-analysis. J Clin Gastroenterol 49: 177-184, 2015.

10 Dabaja BS, Suki D, Pro B, Bonnen M and Ajani J: Adenocarcinoma of the small bowel: presentation, prognostic factors and outcome of 217 patients. Cancer 101: 518-526, 2004

11 Van Cutsem E, Cervantes A, Nordlinger B, Arnold D and Group EGW: Metastatic colorectal cancer: ESMO Clinical Practice Guidelines for diagnosis, treatment and follow-up. Ann Oncol 25(Suppl 3): iii1-9, 2014.

12 Overman MJ, Varadhachary GR, Kopetz S, Adinin R, Lin E, Morris JS, Eng C, Abbruzzese JL and Wolff RA: Phase II study of capecitabine and oxaliplatin for advanced adenocarcinoma of the small bowel and ampulla of Vater. J Clin Oncol 27: 25982603, 2009.

13 Zaanan A, Costes L, Gauthier M, Malka D, Locher C, Mitry E, Tougeron D, Lecomte T, Gornet JM, Sobhani I, Moulin V, Afchain P, Taieb J, Bonnetain F and Aparicio T: Chemotherapy of advanced small-bowel adenocarcinoma: a multicenter AGEO study. Ann Oncol 21: 1786-1793, 2010.

14 Tsushima T, Taguri M, Honma Y, Takahashi H, Ueda S, Nishina T, Kawai H, Kato S, Suenaga M, Tamura F, Morita S and Boku $\mathrm{N}$ : Multicenter retrospective study of 132 patients with unresectable small bowel adenocarcinoma treated with chemotherapy. Oncologist 17: 1163-1170, 2012.

15 Edge SB, Byrd DR, Compton CC, Fritz AG, Greene FL and Trotti A (eds.): AJCC Cancer Staging Manual (7th ed). New York, NY: Springer; 2010.

16 Serour F, Dona G and Birkenfeld S: Primary neoplasms of the small bowel. J Surg Oncol 49: 29-34,1992.

17 Siegel R, Naishadham D and Jemal A: Cancer statistics, 2012. CA Cancer J Clin 62: 10-29, 2012.
18 Cancer Registry and Statistics: Cancer Information Service, National Cancer Center, Japan.

19 Haselkorn T, Whittemore AS and Lilienfeld DE: Incidence of small bowel cancer in the United States and worldwide: geographic, temporal and racial differences. Cancer Causes Control 16: 781-787, 2005.

20 Howe JR, LH K and HR M: The American College of Surgeons Commission on Cancer and the American Cancer Society. Adenocarcinoma of the small bowel: review of the National Cancer Data Base 1985-1995. Cancer 86: 2693-2706, 1999.

21 Xiang XJ, Liu YW, Zhang L, Qiu F, Yu F, Zhan ZY, Feng M, Yan J, Zhao JG and Xiong JP: A phase II study of modified FOLFOX as first-line chemotherapy in advanced small bowel adenocarcinoma. Anticancer Drugs 23: 561-566, 2012.

22 Ecker BL, McMillan MT, Datta J, Mamtani R, Giantonio BJ, Dempsey DT, Fraker DL, Drebin JA, Karakousis GC and Roses RE: Efficacy of adjuvant chemotherapy for small bowel adenocarcinoma: A propensity score-matched analysis. Cancer 122: 693-701, 2016.

23 Gibson Cah MK, Kvols LK and Haller D: Phase II Study of 5fluorouracil, doxorubicin and mitomycin $\mathrm{C}$ for metastatic small bowel adenocarcinoma. Oncologist Gastrointest Cancer 10: 132137, 2005.

24 Raghav K and Overman MJ: Small bowel adenocarcinomasexisting evidence and evolving paradigms. Nat Rev Clin Oncol 10: 534-544, 2013.

25 Overman MJ, Kopetz S, Wen S, Hoff PM, Fogelman D, Morris J, Abbruzzese JL, Ajani JA and Wolff RA: Chemotherapy with 5-fluorouracil and a platinum compound improves outcomes in metastatic small bowel adenocarcinoma. Cancer 113: 2038-2045, 2008.

Received December 30, 2016

Revised February 8, 2017

Accepted February 13, 2017 